\title{
Patient-specific management of severe TBI depending on age and cerebrovascular autoregulation status
}

Vytautas Petkus ${ }^{1}$, Aidanas Preiksaitis ${ }^{1,2,3,4}$, Edvinas Chaleckas ${ }^{1}$, Solventa Krakauskaite ${ }^{1}$, Erika Zubaviciute ${ }^{2}$, Saulius Vosylius ${ }^{2,3}$, Saulius Rocka ${ }^{2,3}$, Daiva Rastenyte ${ }^{4}$, Arminas Ragauskas ${ }^{1}$

${ }^{1}$ Health Telematics Science Institute, Kaunas University of Technology, Lithuania

2 Vilnius University, Faculty of Medicine, Clinic of Neurology and Neurosurgery, Vilnius, Lithuania

${ }^{3}$ Republic Vilnius University Hospital, Department of Neurosurgery, Vilnius, Lithuania

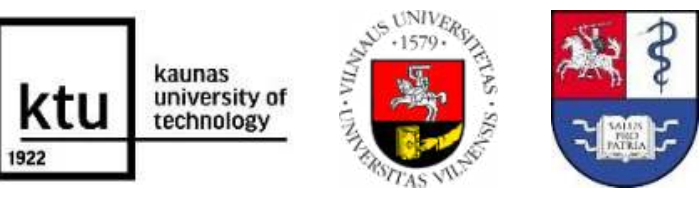

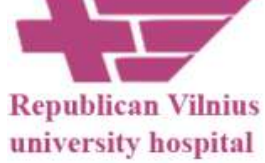

${ }^{4}$ Lithuanian University of Health Sciences, Academy of Medicine, Department of Neurology, Kaunas, Lithuania,

Contact information: Barsausko St. 59 A553-A561, LT-51423, Kaunas, Lithuania, E-mail: telematics@ktu.It

\section{Background}

Identification of patient-specific therapy targets is crucial for traumatic brain injury (TBI) patients. The objectives were to compare the effectiveness of optimal cerebral perfusion pressure (CPPopt) targeted therapy in younger ( $<45$ years) and elderly $(\geq 45$ years) TBI patients as well as to explore the influence of various factors (cerebrovascular autoregulation (CA), cerebral perfusion pressure (CPP) declination from optimal CPP value ( $\triangle$ CPPopt), age, glucose concentration in blood, etc.) on TBI patients' outcome.

\section{Materials and methods}

Multimodal ABP, ICP, CPP and CA monitoring of $81 \mathrm{TBI}$ patients has been performed in Republic Vilnius University Hospital (Lithuania). ICM+ software (Cambridge, UK) was used for continuous CA status assessment by calculating Pressure Reactivity Index (PRx) and for identification of patient-specific CPPopt. The post-hoc analysis of associations between the patients' outcome and complex influence monitored parameters was performed by determining the most important factors affecting outcome.

\section{Results}

The multifactorial logistic regression model showed that the most significant factors affecting TB patient's outcome are age, glucose level in blood serum samples (determined within $24 \mathrm{~h}$ after admission), Helsinki CT (HCT) score, $\triangle$ CPPopt and duration of longest CA impairment when PRx $(\mathrm{t})>0.5$ within 24 hours after admission. The modelled accuracies for unfavorable outcome prediction were $83 \%$ and $88 \%$ respectively.

The age $>45$ years $(P=0.018, \chi 2=15.50)$ was found statistically significantly associated with unfavorable outcome. OptCPP-targeted management was found statistically significantly associated with better outcome for younger patients (age $<45$ years). The critical values of averaged $\Delta$ CPPopt $<-5 \mathrm{mmHg}$ averaged value of $P R x>0.36$ and duration of $L C A I>100 \min (P=0.043, \chi 2=18.00)$ were found associated with mortality for younger patients group ( $<45$ years) (Fig. 1$)$. The critical values of averaged $P R x>0.26$ $(P=0.005, \chi 2=7.80)$ and duration of LCAI $>61 \mathrm{~min}(P=0.019, \chi 2=5.44)$ were founded associated with mortality for elderly patients group ( $>45$ years). However, no statistical differences were found between survivals and not survivals comparing $\triangle \mathrm{CPP}$ opt for elderly patients group (Fig. 1c).

\section{Conclusions}

The main influential factors affecting severe TBI treatment outcomes are patient age, HCT, blood glucose level, and the CA status during the first day after patient admission. CPPopt-targeted patientspecific treatment is useful for stabilizing CA in severe TBI patients, as well as especially beneficial for improving outcome for younger patients less than 45-years-old. CA monitoring in first 24 hours seems to be important because especially in older patients because impaired CA is less long tolerated. However additional research is needed to prove the applicability of CPPopt-targeted therapy for elderly patients greater than 45-years-old.

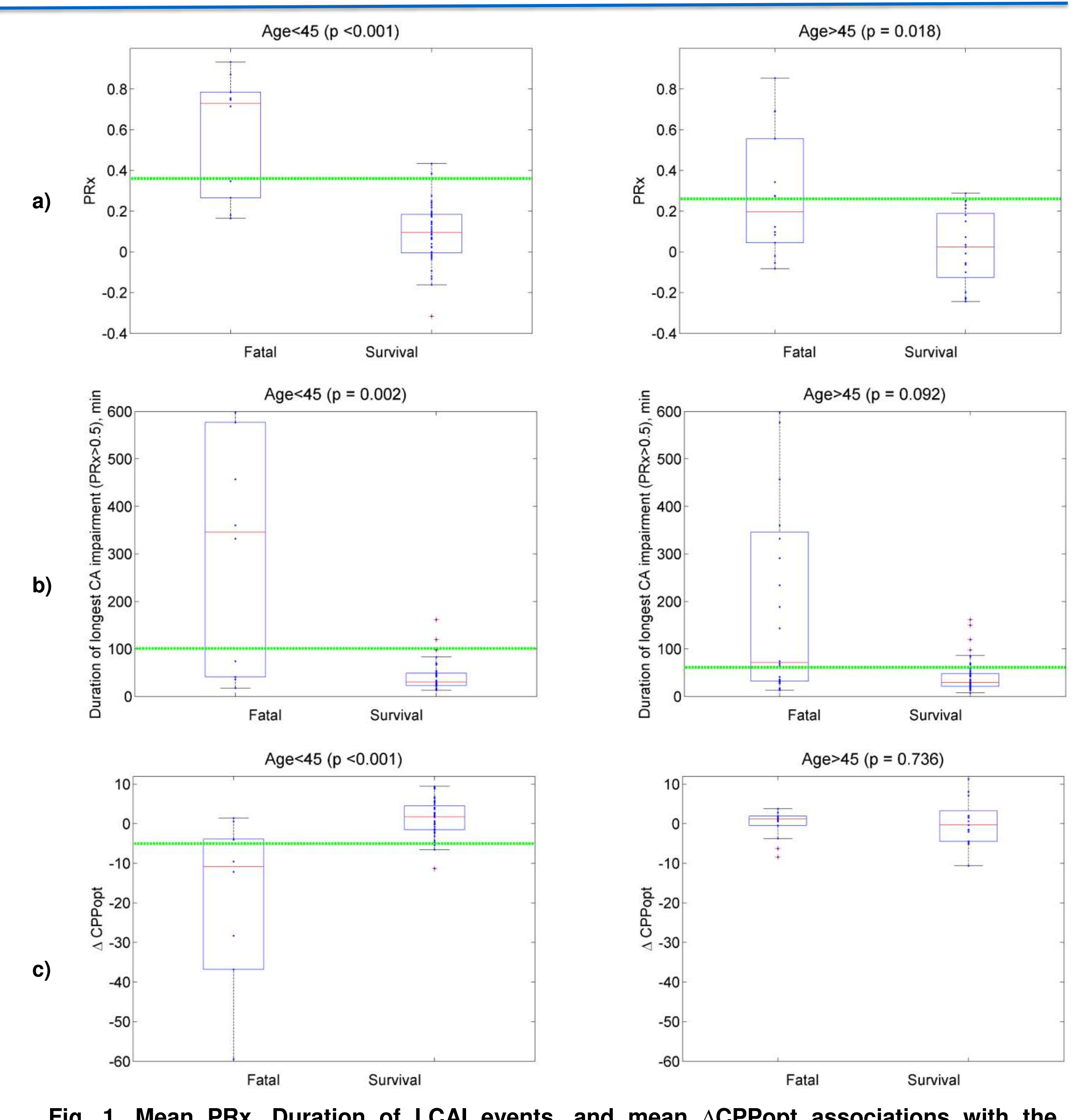

Fig. 1. Mean PRx, Duration of LCAl events, and mean $\triangle$ CPPopt associations with the outcome of younger (age $<45$ years) and elderly (age $>45$ years) TBI patients. Mean PRx above 0.36 and 0.26 and LCAl (PRx $>0.5$ ) longer than $100 \mathrm{~min}$ and $61 \mathrm{~min}$ are associated with fatal outcome for younger and elderly TBI patients, respectively $(a, b)$. Mean $\triangle$ CPPopt below $-5 \mathrm{mmHg}$ is associated with fatal outcome for younger TBI patients. Statistically significant differences of $\triangle$ CPPopt between fatal and non-fatal patients were found only for the younger patients (c). 